\title{
Human Papillomavirus and Cancer
}

\author{
PAMELA HALLQUIST VIALE, RN, MS, CNS, ANP
}

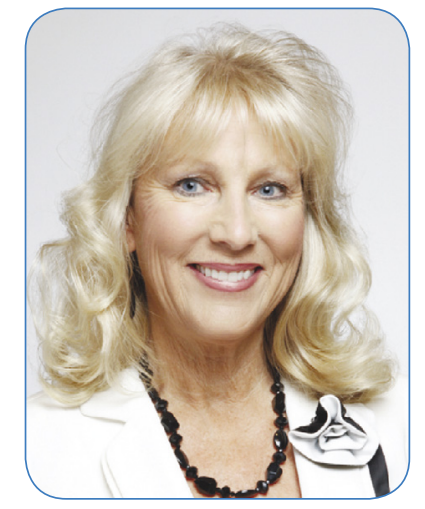

J Adv Pract Oncol 2018;9(7):694-695 https://doi.org/10.6004/jadpro.2018.9.7.1 (c) 2018 Harborside ${ }^{\mathrm{TM}}$

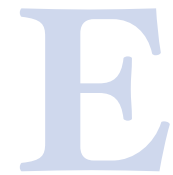

arlier this November, the sixth annual JADPRO Live meeting was held in sunny Hollywood, Florida. The weather was fantastic, we had the most attendees ever (over 1,400), and the sessions were exciting, informative, and relevant to our practice as advanced practitioners! If you weren't able to join us this year, please mark your calendars for next year's meeting, to be held October 24 to 27,2019 , in Seattle. Don't miss it!

\section{HPV VACCINATION}

One of the many sessions I attended was a talk on multimodality therapies for head and neck cancer, presented by Julie Bauman, MD, MPH, and Abby Fuoto, DNP, ANP-BC, AOCNP $^{\circledR}$, ACHPN, both of the University of Arizona Cancer Center. It was a great session that highlighted how human papillomavirus (HPV) 16, which causes half of cervical cancer cases and two thirds of oropharynx cancer, remains a significant health problem. The good news is that we have effective vaccines against this cancer type. What is the bad news?

When quadrivalent HPV recombinant vaccine (Gardasil) was first approved for HPV 6, 11, 16, and 18 in 2006, the news was heralded as a significant breakthrough in the prevention of cervical cancer, genital warts, and anogenital cancer. The vaccine was indicated for girls aged 9 to 26. Since that original approval, a different vaccine, HPV bivalent recombinant vaccine (Cervarix), was approved in 2009 for girls only (covering HPV 16 and 18), and most recently, HPV 9-valent recombinant vaccine (Gardasil 9) for HPV 6, 11, 16, $18,31,33,45,52$, and 58 was approved in 2014 for girls aged 9 to 25 and boys aged 9 to 26 (Bauman \& Fuoto, 2018). In October 2018, Gardasil 9 was approved by the US Food and Drug Administration (FDA) for females and males aged 9 through 45 .

As a nurse practitioner of a certain age, I was not afforded the opportunity to receive the HPV vaccination when it was originally approved. But I can tell you I would have stood in line to receive it if I'd have been able to get it. The FDA stated that HPV vaccination prior to becoming infected with HPV has the potential to prevent more than $90 \%$ of HPV-related cancers from developing at all (US Food and Drug Administration, 2018). However, not all young people are taking advantage of the vaccine for a variety of reasons.

\section{PRESIDENT'S CANCER}

PANEL RECOMMENDATIONS The President's Cancer Panel is committed to increasing HPV vaccine uptake by appropriate patients. Their four goals for increase of the 
vaccine's use include reducing missed clinical opportunities (by concentrated efforts to train providers, engagement of all staff, implementation of standing orders, use of tracking systems, reminders, and improvement of access); increasing parents' acceptance of the HPV vaccine (by education and improved communication of the effectiveness of the vaccine and the safety of the drug, and the appropriate age to receive the vaccine); maximizing access to the HPV vaccine (improving coverage for the vaccination, expanding access to settings for vaccination, and ensuring insurance coverage); and promoting global HPV vaccine uptake (improving vaccination in low and middle income countries by coordination, planning, communication, and education).

The Panel notes that HPV was responsible for almost 630,000 cases of cancer worldwide in 2012; most of these cancers could have been prevented by the vaccines currently available today (President's Cancer Panel, 2018). That is a sobering statistic.

In their excellent presentation at JADPRO Live, Dr. Bauman and Dr. Fuoto noted that HPVrelated throat cancer is an epidemic, with the proportion of cases of HPV-related oropharynx cancer increasing over 20 years from $17 \%$ to an astounding $72 \%$ (Bauman \& Fuoto, 2018). This is largely a preventable cancer. Although generally most cases are curable, not all are, and the treatment is toxic, requiring interdisciplinary therapy and rigorous symptom management. Think how much better off our patients would be if primary prevention were employed instead of treating the tumor afterwards.

\section{ROLE OF \\ ADVANCED PRACTITIONERS}

Advanced practitioners working with the target population for primary prevention with the HPV vaccine should take note of the latest recommendations for improving the numbers of patients receiving the vaccine and the latest FDA approval for use of the vaccine that expanded the ages of appropriate patients. So many cancers cannot be prevented and toxic therapies will be used in the treatment of these patients to improve outcome and effect cure. With an effective prevention strategy for HPV-related cancers, our role is to get the word out and destigmatize the vaccine. Education is one of the things we do very well as advanced practitioners!

\section{References}

Bauman, J., \& Fuoto, A. (2018). Multimodality therapies for head and neck cancer. Presentation given at JADPRO Live 2018, Hollywood, Florida.

President's Cancer Panel. (2018). HPV vaccination for cancer prevention: Progress, opportunities, and a renewed call to action. Retrieved from https://prescancerpanel.cancer.gov/report/hpvupdate/Goal4.html

US Food and Drug Administration. (2018). FDA approves expanded use of Gardasil 9 to include individuals 27 through 45 years old. Retrieved from https://www.fda. gov/NewsEvents/Newsroom/PressAnnouncements/ ucm622715.htm 